\title{
Interpretational factors in conditional reasoning
}

\author{
VALERIE A. THOMPSON \\ University of Saskatchewan, Saskatoon, Saskatchewan, Canada
}

\begin{abstract}
Two experiments examined the role of necessity and sufficiency relationships in conditional reasoning. The results indicated that perceived necessity and sufficiency predicted variability in reasoning performance for four pragmatic relations (permission, obligation, causation, and definition), for both determinant and indeterminant syntactic forms, and for both a conditional arguments and a truth table evaluation task, as well as when the temporal relationship between the antecedent and consequent events was reversed. These data support the general utility of perceived necessity and sufficiency in the interpretation and evaluation of conditional relationships. However, the effects of necessity and sufficiency were smaller for reversed than for forward statements, which suggests that necessity/sufficiency-based interpretations may be more useful for evaluating some types of conditional relations than others. In addition, people were more likely to accept valid rather than invalid arguments, regardless of necessity/sufficiency relations, a finding that suggests that abstract, content-free representations may play a functional role in conditional reasoning.
\end{abstract}

Conditional reasoning entails drawing inferences about situations in which the occurrence of one event is conditional or contingent upon the occurrence of another event. The apparent simplicity of this task is belied by the complexity of the empirical findings regarding it. For example, much recent research has shown that people's inferences are influenced by the content of their premises, even when the logical requirements of the conditional reasoning task are held constant (Byrne, 1989; Cummins, Lubart, Alksnis, \& Rist, 1991; Thompson, in press; Ward, Byrnes, \& Overton, 1990). Moreover, these content effects vary as a function of the task used to measure reasoning, in such a way that content that facilitates performance on one task will not necessarily facilitate performance on another, logically equivalent, task (Markovits \& Savary, 1992; Thompson, in press). These observations highlight the importance of interpretational factors in reasoning and suggest that these factors may interact both with each other and with other aspects of the task environment, in complex and subtle ways.

However, to date, most research has examined the effects of interpretational variables in isolation, without pursuing the question of how these variables may interact. A case in point is the research concerning necessity/sufficiency relations. Although interpretations based

This research was supported by an NSERC postgraduate scholarship to the author, and by NSERC Operating Grant A0087 to Allan Paivio. I wish to acknowledge Jamie Campbell and Peter Denny for several stimulating discussions that helped to refine the ideas presented here, as well as Jamie Campbell, Allan Paivio, Albert Katz, Henry Markovits, and two anonymous reviewers for their helpful comments and suggestions on a previous version of this manuscript. Correspondence concerning this manuscript should be addressed to V. A. Thompson, Department of Psychology, University of Saskatchewan, Saskatoon, SK, Canada S7N 0W0 (e-mail: thompsonv@sask.usask.ca). on perceived necessity and sufficiency have consistently been shown to be important factors in making and evaluating conditional inferences (e.g., Cummins et al., 1991; Digdon, 1986; Markovits, 1984, 1986; Staudenmayer, 1975; Thompson, in press), we do not know how the use of necessity/sufficiency information may be modified by other interpretational variables, such as pragmatic, contextual relations (e.g., Cheng \& Holyoak, 1985) or logical, syntactic relations. Thus, the following experiments examined the role of perceived necessity and sufficiency in the making of inferences about a variety of pragmatic and syntactic relationships.

\section{Conditional Reasoning}

Conditional relationships are often expressed in the generic form "if $p$, then q," where $p$ and $q$ are referred to as the antecedent and consequent, respectively. In a conditional reasoning task, subjects are typically asked to make inferences about the occurrence of one event, given the occurrence or nonoccurrence of the other event. For example, in a conditional arguments task, subjects are asked to indicate the validity of four inferences derived from a conditional statement (e.g., if the car is out of gas, then it stalls). The modus ponens (MP) inference entails concluding q, given p (e.g., the car is out of gas; therefore, it stalls), and modus tollens (MT) entails concluding $\sim p$, given $\sim q$ (e.g., the car has not stalled; therefore, it did not run out of gas). The denying-the-antecedent (DA) and affirming-the-consequent arguments (AC) involve the inference from $\sim \mathrm{p}$ to $\sim \mathrm{q}$ (e.g., if the car does not run out of gas, it will not stall) and from $q$ to $p$ (the car has stalled; therefore, it has run out of gas), respectively.

Logically speaking, the validity of an argument is determined only by its syntactic form; by this criterion, the MP and MT arguments are considered to be valid, and the DA and AC arguments are considered to be fallacies 
(see, e.g., Evans, 1982). However, much research has indicated that people often deviate from this ideal: they frequently make invalid inferences (Evans, 1982; Marcus \& Rips, 1979; Staudenmayer, 1975; Taplin, 1971; Taplin \& Staudenmayer, 1973) and are influenced by both the content of the conditional relationship and the context in which it is presented (Byrne, 1989; Digdon, 1986; Griggs, 1983; Marcus \& Rips, 1979; Markovits, 1984, 1986; Reich \& Ruth, 1982; Rumain, Connell, \& Braine, 1983; Staudenmayer, 1975).

\section{Necessity, Sufficiency, and Conditional Reasoning}

One way to explain these types of content effects is to assume that reasoning is directly mediated by available knowledge of the specific conditional relationship, such as the perceived necessity and sufficiency of the relationship (e.g., Digdon, 1986; Markovits, 1984, 1986; Pollard, 1982; Thompson, in press). Necessary relationships are those for which the consequent event occurs only when the antecedent event occurs (e.g., if the TV is plugged in, then it works). Sufficient relationships are those for which the consequent always occurs when the antecedent occurs (e.g., if the car runs out of gas, then it stalls). Because these approaches assume that information about necessity/sufficiency relations can be cued from either the context or content of the conditional relationship, I will refer to them collectively as contextual cuing theories.

According to this approach, responses to all four logical questions should vary systematically as a function of the perceived necessity and sufficiency of the conditional relationship (Bindra, Clarke, \& Shultz, 1980; Staudenmayer, 1975): the DA and AC inferences should be affected by the necessity of the relationship, whereas the MP and MT inferences should be affected by the sufficiency of the relationship.

Perceived necessity and the AC/DA inferences. According to the contextual cuing theories, people should be more likely to commit the AC and DA "fallacies" when the relationship is perceived to be necessary than when it is not, as illustrated below. For a necessary relationship, q occurs only when $\mathrm{p}$ does; therefore, if $\mathrm{q}$ has happened, it follows that $p$ must also have happened (AC), and if $p$ did not happen, then q could not have happened either. In contrast, when the relationship is not necessary, it is possible for q to occur without $\mathrm{p}$. Thus, the fact that $q$ has occurred is no guarantee that $p$ did also (AC), nor does the absence of $p$ guarantee the absence of $q(D A)$. Consequently, the AC and DA inferences seem less plausible for nonnecessary than for necessary relationships. Consistent with this hypothesis, several investigations have found that people are more likely to make the $\mathrm{AC}$ and DA inferences for necessary than for nonnecessary relations (Bucci, 1978; Digdon, 1986; Markovits, 1984, 1986; Staudenmayer, 1975; Thompson, in press).

\section{Necessary:}

If the TV is plugged in, then it works.
AC: The TV is working. Is it plugged in? (yes)

DA: The TV is not plugged in. Is it working? (no)

Nonnecessary:

If the car runs out of gas, then it stalls.

AC: The car has stalled. Did it run out of gas? (maybe)

DA: The car has not run out of gas. Did it stall? (maybe)

Additional support for this position derives from the observation that factors that underlie judgements of perceived necessity, such as the availability of alternative antecedents, also affect the probability of accepting the DA and AC inferences. Alternative antecedents are conditions other than $p$ that cause $q$ to occur, and thus, the presence of alternative antecedents indicates that $p$ is not necessary for q (Pollard, 1982). For example, in the "car" example above, alternative antecedents would be other ways that a car might stall without running out of gas (e.g., broken fanbelt, overheated engine, etc.).

Several investigations point to a relationship between alternative antecedents and inference patterns. Markovits (1984) demonstrated that subjects who were aware of the possibility of alternative antecedents were less likely to make the DA and AC inferences than those who were not. Similarly, there is an inverse relationship between the number of alternative antecedents cued by a conditional relationship, and the probability that subjects accept the DA and AC inferences (Cummins et al., 1991; Thompson, in press). In addition, when alternative antecedents are provided by the experimenter, subjects are less likely to commit the AC and DA fallacies (Byrne, 1989; Rumain et al., 1983).

Perceived sufficiency and the MP/MT inferences. According to the contextual cuing theories, people should be more likely to "correctly" make the MP and MT inferences when the relationship is sufficient than when it is nonsufficient, as illustrated below. For sufficient relationships, $q$ always happens when $p$ does; thus, if $p$ happens, then so must $q$ (MP), and if q has not happened, it means that $p$ cannot have happened either (MT). However, for nonsufficient relationships, $p$ does not guarantee $q$, and, as a result, neither the MP nor the MT inference seems compelling for nonsufficient relationships.

Sufficient:

If the car runs out of gas, then it stalls.

MP: The car has run out of gas. Will it stall? (yes)

MT: The car has not stalled. Did it run out of gas? (no)

Nonsufficient:

If a person smokes, then he/she gets lung cancer.

MP: A person smokes. Will he/she get lung cancer? (maybe)

MT: A person does not have lung cancer. Does he/she smoke? (maybe)

Although less research has been done to support the role of perceived sufficiency in conditional inferences, the available data support the prediction that subjects are less likely to endorse the MP and MT inferences for non- 
sufficient than for sufficient relations (Thompson, in press). In addition, the acceptability of the MP and MT inferences appears to vary as a function of factors that affect the perceived sufficiency of the rule, such as the availability of alternative consequents. Alternative consequents are situations in which $p$ occurs without $q$, and thus they indicate that the conditional relationship is nonsufficient (i.e., because not everyone who smokes gets lung cancer, smoking is not a sufficient condition for getting lung cancer). Consistent with this hypothesis, two recent studies have found that people are less likely to make the MP and MT inferences when alternative consequents are available than when they are not (Byrne, 1989; Cummins et al., 1991).

\section{The Generality of Necessity/Relations in Conditional Reasoning}

Although there is much evidence to indicate that both necessity/sufficiency relations and the availability of counterexamples influence reasoning in a wide variety of stimulus items and tasks, little is known about how these factors interact with other variables known to affect reasoning. Specifically, can different types of pragmatic (see, e.g., Cheng \& Holyoak, 1985) or syntactic relations either reduce or augment the effects of necessity/sufficiency relations on reasoning performance? Although no concrete evidence is available to answer this question, some researchers have speculated that considerations based on pragmatic or logical principles could override the use of necessity and sufficiency information.

Necessity, sufficiency, and pragmatic relations. Context-based approaches typically assume that reasoning is mediated by a variety of context-specific reasoning rules or heuristics that apply to common inferential situations, such as permission, causation, obligation, etc. (e.g., Cheng \& Holyoak, 1985; Cheng, Holyoak, Nisbett, \& Oliver, 1986; Cosmides, 1989; Gigerenzer \& Hug, 1992; see also Fillenbaum, 1986, for related ideas); these rules capture a pragmatic understanding of concepts such as causation and obligation. For example, context-based approaches often assume that reasoning about the social world is mediated by different assumptions, heuristics, or inferential rules than is reasoning about the physical world. Thus, the inferences that are pragmatic for social regulations, such as promises and threats (Fillenbaum, 1986), cost-benefit transactions (Cosmides, 1989; Gigerenzer \& Hug, 1992), and permission and obligation contexts (Cheng \& Holyoak, 1985; Cheng et al., 1986) may be different from the inferences that are pragmatic for dealing with natural regularities such as contingency or covariation relations, causal relations, and categorical relations.

One implication of these approaches is that the effects of necessity and sufficiency might vary as a function of the pragmatic relationship expressed by the conditional statement. That is, because reasoning may be based on pragmatic principles (e.g., Cheng \& Holyoak, 1985; Cheng et al., 1986; Cosmides, 1989; Markovits \& Savary, 1992), the types of factors that influence reasoning about one pragmatic relation may not influence performance for all such relations. Indeed, Cheng and Holyoak (1985) argued that some physical relationships, such as causal and contingency relations, might be more sensitive to an interpretation based on perceived necessity than would permission relations. According to Cheng and Holyoak (1985), "Since events are sometimes perceived as having a single cause [i.e., they are interpreted as necessary relationships], problems evoking a causal schema are more likely to lead to the fallacy of Affirming the Consequent than problems evoking the permission schema" (p. 398). Thus, whereas previous researchers have demonstrated that reasoning about causal situations (Cummins et al., 1991; Staudenmayer, 1975) or about categorical relationships (Bucci, 1978) is influenced by necessity/ sufficiency relationships, to what extent these factors operate for social regulation statements is not clear, nor whether they operate at all.

Cummins et al. (1991) proposed a second reason for why the effects of necessity/sufficiency relations might vary across pragmatic contexts. They argued that the types of counterexamples that are available will differ from one pragmatic relationship to another. In the case of causal relationships, for example, they defined alternative antecedents to be alternative causes (other events that will produce the effect) and alternative consequents to be disabling conditions (events that might prevent the effect from occurring). Neither type of counterexample would be relevant to other pragmatic relations. For example, social regulation statements express rules concerning things one should and should not do; counterexamples to these rules are more likely to consist of reasons for why people might violate the rule. Thus, because there may be qualitative differences among the counterexamples elicited by different contexts, it is possible that the manner in which they are evaluated may also differ, in such a way that necessity/sufficiency relations would have different effects across pragmatic contexts.

In summary, necessity/sufficiency relations may be important to the interpretation of some pragmatic relations, but not others. To test this hypothesis, the following experiments examined reasoning performance for four pragmatic relations (causation, definition, permission, and obligation); for each pragmatic relation, necessity/sufficiency relations were varied orthogonally.

Necessity, sufficiency, and syntax. In logic, syntax refers to the form of an argument, independent of its content. For example, modus tollens is an inference from the absence of $q$ to the absence of $p$, regardless of whatever specific concrete entities $p$ and $q$ refer to. Typical conditional reasoning tasks make use of four syntactic forms, two of which lead to valid conclusions (i.e., the MP and MT forms), and two of which yield no valid conclusions (i.e., the AC and DA forms). The second purpose of the following experiments was to determine whether necessity/sufficiency-based interpretations are important regardless of the syntactic form of the question that is asked.

To answer this question, one needs to manipulate necessity/sufficiency relations independently of the syn- 
tactic form of the question that is used to measure their effects. In the present experiments, this was accomplished by reversing the antecedent and consequent clauses in the conditional statements. Because reversing a conditional relationship changes the syntactic form of the logical questions, but not the content of the questions themselves, this procedure allows one to evaluate the effects of content independently of the effects of syntax, as the example below illustrates.

\section{Forward: \\ If the car is out of gas, then it stalls. MP: The car is out of gas. Will it stall? (yes) \\ DA: The car is not out of gas. Will it stall? (maybe) \\ $\mathrm{AC}$ : The car has stalled. Is it out of gas? (maybe) \\ MT: The car has not stalled. Is it out of gas? (no) \\ Reversed: \\ If the car has stalled, then it is out of gas. \\ MP: The car has stalled. Is it out of gas? (maybe) \\ DA: The car has not stalled. Is it out of gas? (no) \\ AC: The car is out of gas. Will it stall? (yes) \\ MT: The car is not out of gas. Will it stall? (maybe)}

As the example illustrates, the same question (e.g., the car has not stalled, is it out of gas?) plays different syntactic roles for the forward and reversed statements. For the forward statement, this question is the MT question, but for the reversed statement, it is the DA question. Similarly, the content of the forward MP, AC, and DA questions corresponds to the content of the reverse $\mathrm{AC}$, $\mathrm{MP}$, and MT questions.

It follows, therefore, that if necessity/sufficiency-based interpretations operate independently of syntax, then the effects of necessity and sufficiency should be similar for both forward and reversed forms. Responses to the forward MP and MT questions should vary as a function of the perceived sufficiency of the conditional relationship, as should responses to the reversed $\mathrm{AC}$ and $\mathrm{DA}$ queries. In addition, responses to the forward $A C$ and $D A$ questions, as well as their reversed MP and MT counterparts, should be mediated by the perceived necessity of the conditional relationship. Thus, to the extent that content effects are independent of syntax, same-content questions should elicit the same responses, regardless of the syntactic form of the questions.

Conversely, to the extent that syntactic relations influence reasoning independently of content, one would expect subjects to be influenced by the syntactic relationship expressed by the sentence. For example, one might expect subjects to give more "yes" and "no" responses to the forward MP and MT questions (where it is logically correct to do so) than to their reversed AC and DA counterparts (where it is logically fallacious to do so). Similarly, one might expect to observe more "maybe" responses to the forward $\mathrm{AC}$ and DA questions than to their reversed MP and MT counterparts, because the former response is logically correct while the latter is not.

\section{Summary}

It is possible that other interpretational and logical factors, such as pragmatic and syntactic relations, could override interpretations based on perceived necessity and sufficiency. Thus, the purpose of the following two experiments was to determine whether the predicted effects of necessity/sufficiency relations would be observed for both social and physical relations, as well as for both determinant and indeterminant syntactic forms. This was accomplished by varying necessity/sufficiency relations independently of both pragmatic relation and syntactic form.

\section{EXPERIMENT 1}

In Experiment 1, reasoning performance was evaluated in a conditional arguments task. For this task, subjects were told to evaluate the four logical questions (MP, DA, AC, and MT) by responding "yes," "no," or "maybe" to each question. Each subject made inferences about 32 conditional relationships, which were chosen for inclusion in this study on the basis of previously collected normative ratings. The statements were selected to vary orthogonally with respect to necessity, sufficiency, and pragmatic relationships, and they were presented in both forward and reversed form.

\section{Method}

\section{Subjects}

Forty undergraduate students from the University of Western Ontario completed the arguments task to fulfill a requirement in an introductory psychology course.

\section{Materials}

The items used in this experiment consisted of 32 forward conditional statements and their reversed counterparts. In all the forward sentences, $p$ was the temporally prior event; in the reverse statements, the order of the $p$ and $q$ clauses was reversed so that $q$ was the temporally prior event. These items instantiated two types of social regulation contexts (permission and obligation) and two natural regularities (categorical and causal relations). For each pragmatic relation, an equal number of sentences represented each possible necessity by sufficiency combination.

These items were selected from a larger pool of 111 items for which normative data was available. A brief summary of the procedure used to collect the normative data is presented below; complete details are available from the author upon request. Two groups of subjects participated in the normative study: one group evaluated necessity/sufficiency relations, and the other evaluated pragmatic, contextual relations. Each item in the normative pool was evaluated by between 28 and 30 subjects.

For the necessity/sufficiency (NS) ratings, subjects were asked to indicate, on a 7-point scale, how necessary and sufficient the antecedent was to the consequent. The ratings questions all followed the generic pattern illustrated below:

It is necessary for " $p$ " to happen in order for " $q$ " to happen.

" $P$ " happening is enough to ensure that " $q$ " will happen.

For this study, items were chosen whose mean ratings differed from the overall mean by at least one standard deviation. In the few cases in which it was not possible to meet this criterion, such as the nonnecessary and sufficient $(-\mathrm{N}+\mathrm{S})$ permission and obligation statements, and the nonnecessary and nonsufficient $(-\mathrm{N}-\mathrm{S})$ permission statements, sentences were chosen that were as close as possible to the criterion boundary.

A second group of subjects was asked to determine the most appropriate pragmatic interpretation for each item. To do so, they 
were asked to indicate which of the following definitions best described the relationship expressed in the sentence.

Obligation. Obligation statements describe a situation or circumstance that creates some degree of obligation to undertake an action.

Permission. Permission statements describe a prerequisite or a criterion that a person satisfies before undertaking a course of action.

Causal. Causal statements describe a situation in which one event causes another to occur.

Definition. Definition statements describe features that characterize membership in a particular category.

Sentences with the highest overall rate of agreement were chosen for inclusion in the study; for the 32 forward items, the mean agreement was $85 \%$. However, the category agreement for the reversed forms of these sentences was considerably lower (72\%); consequently, only the forward sentences were used to determine whether the effects of necessity and sufficiency generalize across pragmatic relations.

The 32 statements selected appear in Appendix A. There were 8 statements representing each necessity by sufficiency combination; of these 8,2 represented each pragmatic relationship. ${ }^{1}$

\section{Procedure for the Reasoning Task}

Each of the 40 subjects received all 32 reasoning problems, presented 7 to a page. Each problem consisted of an underlined conditional sentence followed by four logical questions (MP, MT, AC, and DA). ${ }^{2}$ Following each question was a space where the subject was to indicate his/her response: "yes," "no," or "maybe." The order of the four logical questions was randomly determined on every trial.

For every subject, half the sentences were presented in forward form, and the other half were presented in reversed form. The sentences were divided so that each subject received one forward and one reversed sentence in each necessity/sufficiency $\times$ pragmatic relationship cell. The subjects were "yoked" so that the sentences presented to the 1st subject in forward form would be presented to the 2 nd subject in reversed form. For each pair of subjects, the sentences that were to be presented in forward and reversed form were chosen randomly, and the order in which the problems were presented was random across subjects.

The instructions explained that the purpose of the study was to examine deductive reasoning. The subjects were told to answer the questions only on the basis of conclusions that followed logically from the original statement. They were told to answer each question with a "yes," a "no," or a "maybe." The "maybe" response was to be used if the answer to the question could be either "yes" or "no," on the basis of the information provided. The subjects were given as much time as was necessary to complete the questionnaire (approximately $20 \mathrm{~min}$ ).

\section{Results and Discussion}

The effects of necessity and sufficiency on reasoning performance were examined in two separate sets of analyses. The first set addressed the issue of how interpretations based on perceived necessity and sufficiency generalized across pragmatic relations. As mentioned previously, the classification agreement scores were low for the reversed form of the statements; thus, the pragmatic relationship variable was analyzed only for the forward conditionals. The second set compared forward and reversed statements in order to determine the relative contributions of syntactic form and content (i.e., necessity/sufficiency relations) to performance on the conditional arguments task.

\section{Forward Sentences: Necessity and Sufficiency by Pragmatic Relation}

The responses to the four logical questions for the forward statements are summarized in Figures 1 and 2. These responses are scored in relation to the "correct" pattern; thus, Figure 1 represents "yes" and "no" responses to the MP and MT questions, and Figure 2 represents "maybe" responses to the DA and AC questions. These data were analyzed with three-factor within-subjects analyses of variance (ANOVAs); the source tables for these analyses are reported in Appendix B. For these and all subsequent ANOVAs, the degrees of freedom for the analysis were adjusted using the Geisser-Greenhouse correction whenever the assumption of sphericity in the variance-covariance matrix was not met.

As is apparent from Appendix B, the three-way interaction was significant for each of the logical questions. Thus, four separate two-factor (necessity $\times$ sufficiency)
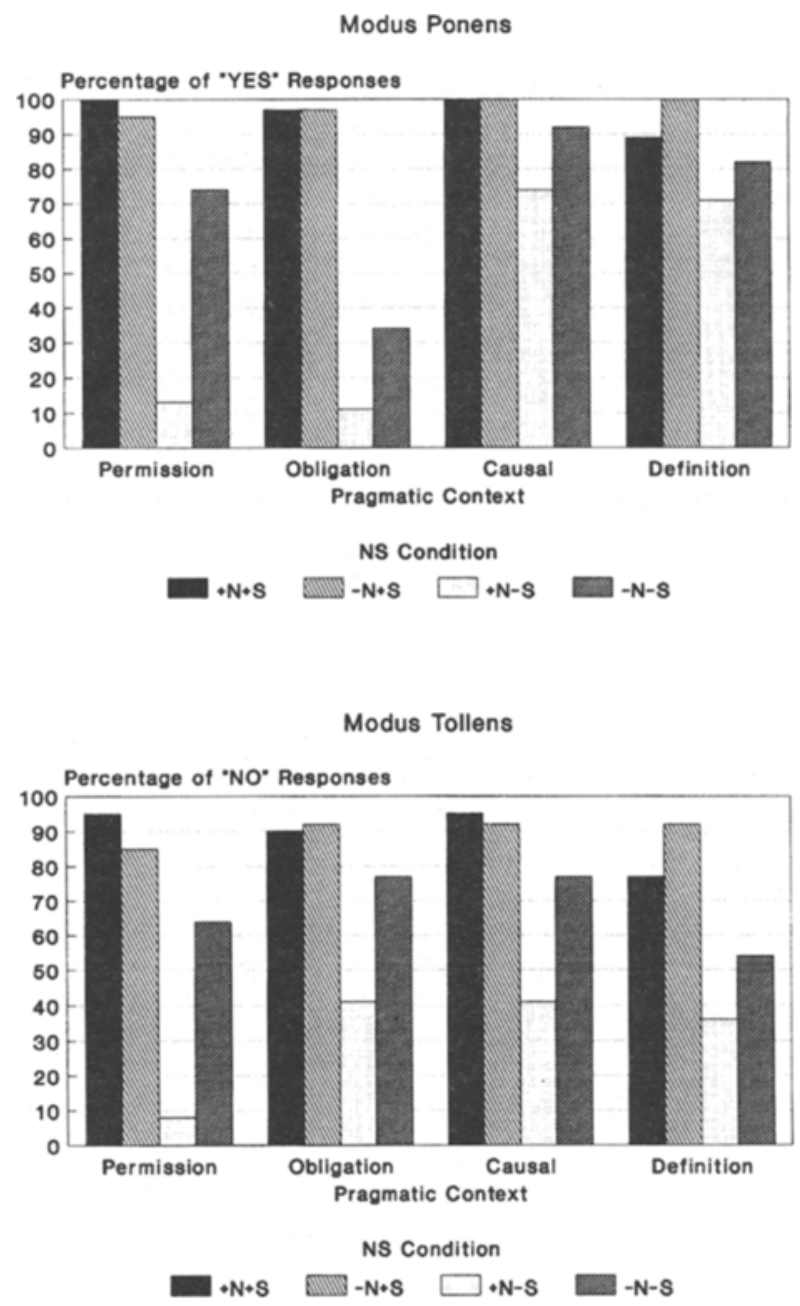

Figure 1. Percentage of determinant ("yes" and "no") responses given to the modus ponens (MP) and modus tollens (MT) questions, as a function of necessity, sufficiency, and pragmatic relation. NS, necessity/sufficiency: $+N$, necessary; $+S$, sufficient; $-N$, nonnecessary; $-\mathbf{S}$, nonsufficient. 
Affirming the Consequent

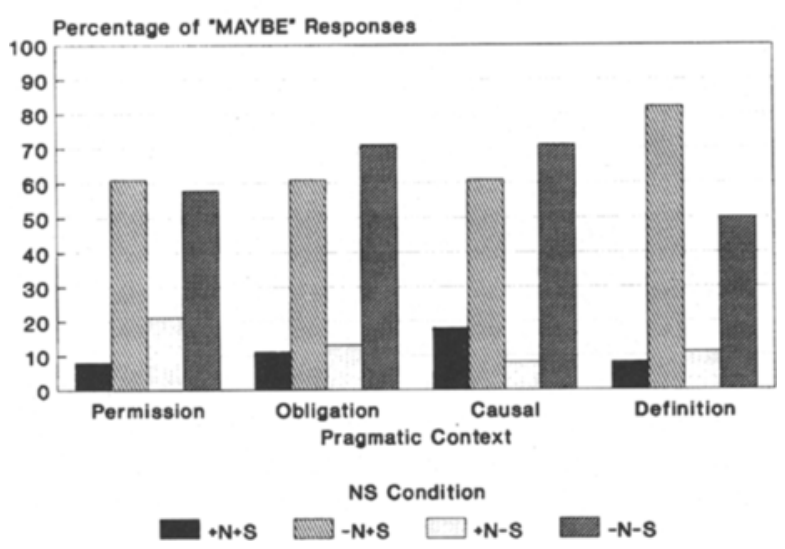

Denying the Antecedent

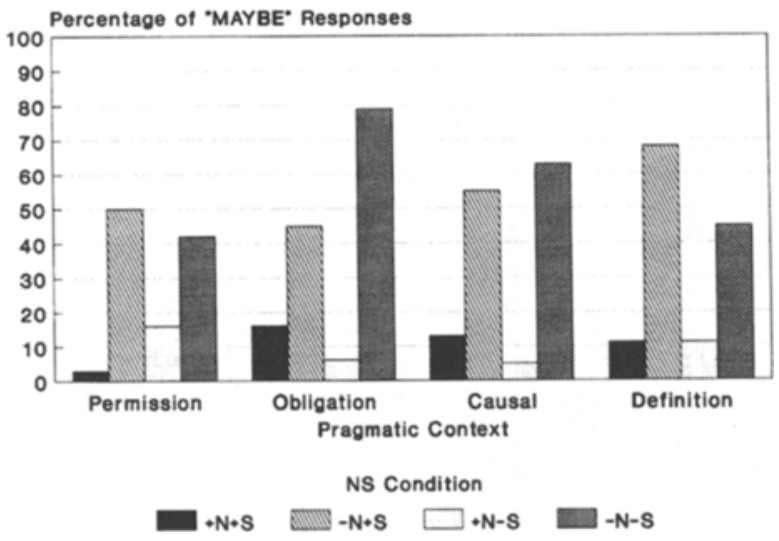

Figure 2. Percentage of indeterminant ("maybe") responses given to the affirming-the-consequent and denying-the-antecedent questions, as a function of necessity, sufficiency, and pragmatic relation. $\mathbf{N S}$, necessity/sufficiency: $+\mathbf{N}$, necessary; $+\mathbf{S}$, sufficient; $-\mathbf{N}$, nonnecessary; - $S$, nónsufficient.

analyses were conducted in order to determine whether the predicted effects of necessity and sufficiency would be observed for all four pragmatic relations. To simplify the reporting of the analyses, the analyses of the MP and MT questions will be presented together here, as will the analyses of the DA and AC questions.

Responses to the MP and MT questions. For the MP and MT responses, the contextual cuing theories predicted that more "yes" and "no" responses should be given to sufficient than to nonsufficient relationships. As is apparent in Figure 1, this general pattern was observed; statistical analyses also supported this prediction. Two-factor (necessity $\times$ sufficiency) within-subjects ANOVAs of the responses within each pragmatic context revealed that there was a main effect of sufficiency for both MP and MT questions for all pragmatic relations $[F(1,39) \geq 10.64, p \leq .002]$. These findings confirm that the predicted effects of sufficiency on condi- tional reasoning performance were observed for all the pragmatic relationships examined here.

In addition, however, there were two nonpredicted findings. For all pragmatic relations, there was a significant main effect of necessity $[F(1,39) \geq 5.05, p \leq .031]$, and for all but the definition statements, there was a significant interaction between necessity and sufficiency $[F(1,39) \geq 6.65, p \leq .003]$. Post hoc tests (NewmanKeuls, $\alpha=.05$ ) indicated that these interaction effects were attributable to the effects of one cell: for all pragmatic relations, there were fewer determinant ("yes" and "no") responses to the MP and MT questions in the necessary and nonsufficient $(+\mathrm{N}-\mathrm{S})$ condition than in any of the other NS conditions.

These findings indicate that some unanticipated aspect of the items in the $-\mathrm{S}$ conditions increased the perceived sufficiency of the $-\mathrm{N}-\mathrm{S}$ statements relative to the $+\mathrm{N}-\mathrm{S}$ statements. One hypothesis has to do with the fact that the $-\mathrm{N}-\mathrm{S}$-statements were the only ones used that expressed a noncontingent relationship between $p$ and q. Because the "if then" syntax usually denotes some type of contingency between $p$ and $q$, subjects may have had difficulty making an appropriate interpretation of the $-\mathrm{N}-\mathrm{S}$ statements. To provide a sensible interpretation, they may have settled on a default interpretation consistent with the formal meaning of "if then"-namely, that $q$ is contingent upon $p$. Under this interpretation, $q$ always occurs when $p$ does, and therefore, such an interpretation would lead to determinant responses to the MP and MT questions. Thus, if subjects were more likely to use the default interpretation of "if then" for the $-\mathrm{N}-\mathrm{S}$ statements than for the $+\mathrm{N}-\mathrm{S}$ statements, there would be more determinant responses on the MP and MT questions for the former than for the latter.

Responses to the DA and AC questions. As was the case with the MP and MT questions, the three-factor interaction was significant for both the $\mathrm{DA}$ and $\mathrm{AC}$ questions. Consequently, the effects of necessity and sufficiency were examined individually for each pragmatic relation. For both questions, the expected main effect of necessity emerged for all four pragmatic relations $[F(1,39) \geq 34.20, p<.001]$. As depicted in Figure 2, this finding is clearly interpreted to mean that more "maybe" responses were given to these questions for nonnecessary than for necessary relations. Thus, the predicted effects of perceived necessity emerged for all of the pragmatic relations tested here.

In addition, for some of the pragmatic relations, there was a significant interaction between necessity and sufficiency. Specifically, for the DA question, the necessity $X$ sufficiency interaction was significant for the definition and obligation statements $[F(1,39) \geq 5.01, p \leq$ $.03]$; for the AC questions, it was significant for the causal and definition questions $[F(1,39) \geq 5.03, p \leq$ $.03]$. Post hoc tests (Newman-Keuls, $\alpha=.05$ ) revealed that the form of this interaction varied across pragmatic relations. For example, in the case of the DA question, there were more "maybe" responses given to the $-\mathrm{N}+\mathrm{S}$ definition statements than to the $-\mathrm{N}-\mathrm{S}$ statements, but 
the direction of this difference was reversed for the obligation statements. In all, there did not appear to be a consistent pattern to these interaction effects.

Summary. Overall, necessity and sufficiency predicted responses to all four logical questions. Responses to the MP and MT questions varied as a function of the sufficiency of the conditional relationship, and responses to the DA and AC questions varied as a function of the necessity of the relationship. Moreover, this pattern was observed for all of the pragmatic relations examined here, a finding that supports the conclusion that necessity and sufficiency are interpretational variables whose effects generalize across a range of pragmatic, contextual relations.

\section{Reversed Sentences: Necessity, Sufficiency, and Syntactic Form}

A comparison for forward and reversed sentences provided an opportunity to examine the effects of necessity and sufficiency on reasoning independently of the effects of the syntactic form of the question. This is because the logical questions for the reversed sentences had the same content as the forward sentences had, but they had different syntactic structures. Specifically, the MP and MT questions for the forward problems had the same content as did the $\mathrm{AC}$ and DA questions for the reversed problems. Similarly, the forward AC and DA questions had the same content as did the reversed MP and MT questions. Therefore, to the extent that responses are mediated by content, one would expect the same responses to have been given to the forward questions and their reversed counterparts. In contrast, to the extent that responses are mediated by syntactic form, subjects should have been more likely to give determinant (yes and no) responses to the MP and MT questions than to their AC and DA counterparts, because "yes" and "no" responses are logically correct only for the MP and MT questions. The response patterns for the forward and reversed statements are summarized as a function of necessity/sufficiency (NS) relations in Figure 3; questions having the same content, but different syntactic forms, are vertically aligned.

Responses to the forward MP/MT and reverse AC/DA questions. The first set of analyses compared the proportion of determinant ("yes" and "no") responses made for the forward MP and MT questions to their reversed AC and DA counterparts using two three-factor (necessity $X$ sufficiency $\times$ syntactic form) within-subjects ANOVAs (source tables for the ANOVAs are presented in Appendix B). Because the three-factor interaction was significant for the MP/AC analysis, but not the MT/DA analysis, these analyses are discussed separately.

The analysis of the forward MT questions and their reversed DA counterparts permitted a straightforward interpretation of the findings. As predicted, there was a significant main effect of sufficiency, which indicated that there were more determinant responses to these questions for sufficient than for nonsufficient relations. Because sufficiency did not interact with syntactic form, it would appear that the difference between sufficient and nonsufficient statement was constant for both the MT and DA syntax. In addition, there was an interaction between necessity and sufficiency. This interaction had the same form as it did in the previous section-namely, there were fewer determinant responses made in the $+\mathrm{N}-\mathrm{S}$ condition than in the $-\mathrm{N}-\mathrm{S}$ condition. Again, there was no higher order interaction with syntactic form, indicating that this effect was similar for both the forward MT and the reversed DA questions.

The analysis of the forward MP/reversed AC questions was somewhat more complex, because in addition to the effects obtained for the MT/DA analysis, there was also a significant three-factor interaction between necessity, sufficiency, and syntactic form. This indicated that differences among NS conditions varied for the MP and $\mathrm{AC}$ questions. However, the presence of ceiling effects for the forward MP responses make the interpretation of this interaction difficult. Specifically, the difference between sufficient and nonsufficient forms may

Forward Statements

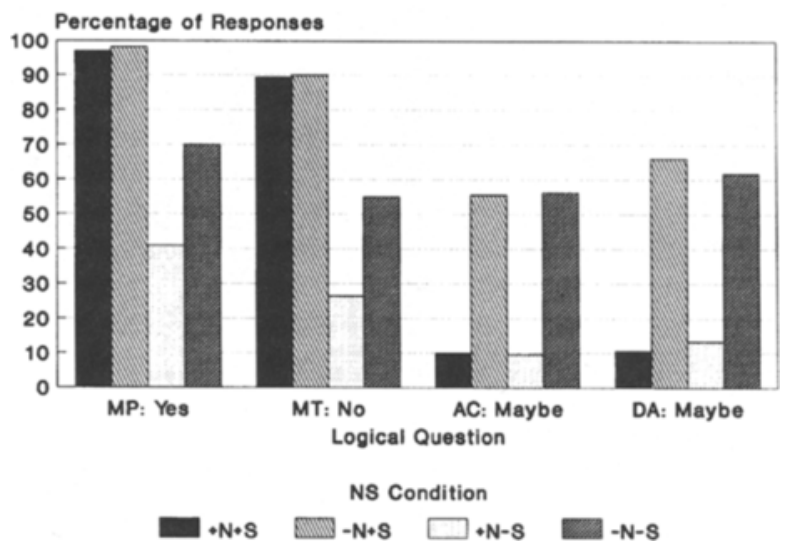

Reverse Statements

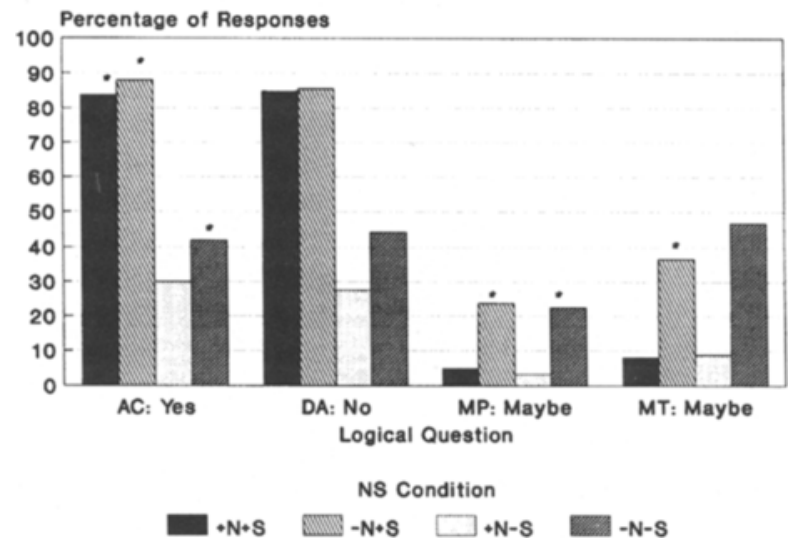

Figure 3. Responses to the logical questions, as a function of necessity, sufficiency, and syntactic form. NS, necessity/sufficiency: $+N$, necessary; $+S$, sufficient; $-\mathbf{N}$, nonnecessary; $-\mathbf{S}$, nonsufficient. 
have been smaller for the MP question than the AC question $(42 \%$ vs. $50 \%)$ because of the effects of syntax, or because ceiling effects prevented a larger range for the MP question. Finally, there was a main effect of syntactic form in this analysis. Because this factor interacted with necessity and sufficiency, paired-difference $t$ tests compared MP and AC responses in each of the four NS conditions $(\alpha=.05 / 4)$. Significant differences between the two syntactic forms are marked with asterisks in Figure 3. Although not all the comparisons were significant, the significant comparisons were all in the predicted direction: subjects were more likely to give "yes" responses to the MP questions, where it was logically correct to do so, than to the $\mathrm{AC}$ questions, where it was not.

In summary, perceived sufficiency affected responses regardless of the syntactic form of the question: subjects gave more determinant responses to sufficient than to nonsufficient relations. In contrast, the evidence regarding the effects of syntactic form was mixed; there was no effect of syntactic form in the MT/DA analysis, but there were some effects in the MP/AC analysis. Thus, there was some evidence to suggest that responses to the logical questions were mediated by syntactic, logical relationships.

Responses to the forward AC/DA and the reverse MP/MT questions. The second set of analyses compared the percentage of "maybe" responses given to the forward AC and DA questions to their reversed MP and MT counterparts (source tables for the ANOVAs are presented in Appendix B). The three-factor interaction between syntactic form, necessity, and sufficiency was significant for both the AC/MP and DA/MT analyses. Again, however, caution must be exercised in interpreting this interaction, this time because of floor effects for the reversed MP and MT questions.

The prediction derived from the contextual cuing theories-namely, that responses to all four questions should vary as a function of perceived necessity -was confirmed by a significant main effect of necessity in both the AC/MP and the DA/MT analyses. Because of the three-factor interaction, separate analyses of necessity and sufficiency were performed for each of the logical questions; the main effect of necessity emerged for all four questions $[F(1,39) \geq 27.06, p<.001]$.

In addition, there was a main effect of syntactic form for both analyses. Paired-difference $t$ tests $(\alpha=.05 / 4)$ were computed to compare "maybe" responses to the AC and DA questions to their reversed MP and MT counterparts within each of the four NS conditions; as before, significant differences are represented by asterisks in Figure 3. Again, however, because of the floor effects, caution must be exercised in interpreting the presence and absence of differences. Where the differences were reliable, they indicated that subjects were less likely to give indeterminant (maybe) responses to the reversed $\mathrm{MP}$ and MT problems than to their forward $\mathrm{AC}$ and DA counterparts. In other words, subjects were more likely to give indeterminant responses when it was logically correct to do so (for the DA and $\mathrm{AC}$ questions) than when it was not (for the MP and MT questions).

\section{Conclusions}

Necessity/sufficiency relations were found to be important for interpreting both social and physical regulations; and the effects of perceived necessity/sufficiency were observed for all four logical questions, as well as for the forward and reversed forms. These data indicate that necessity/sufficiency relations are of general utility in the interpretion of conditional relations. In addition, some evidence was obtained which suggested that responses were influenced by syntactic relations in such a way that questions that had the same content, but different syntactic relations, elicited different responses. This sensitivity to syntactic form suggests a role for contentfree inferential processes (see, e.g., Braine \& O'Brien, 1991; Johnson-Laird, Byrne, \& Shaeken, 1992) in making and evaluating conditional inferences.

\section{EXPERIMENT 2}

The purpose of this experiment was to extend the general utility of the necessity/sufficiency approach by replicating the findings of Experiment 1 using the truthtable evaluation task (Evans, 1983). The truth-table evaluation task requires subjects to determine which of the four truth-table contingencies ( $p \& q, p \& \sim q, \sim p \& q, \sim p$ $\& \sim q)$ are inconsistent with the conditional rule. For example, subjects would be asked to indicate which of the following four contingencies was inconsistent with the rule: "if the car is out of gas, then it will stall."

$$
\begin{aligned}
& \text { The car is out of gas and it stalls. (p \& q) } \\
& \text { The car is out of gas and it does not stall. (p \& } \sim q) \\
& \text { The car is not out of gas and it stalls. ( p \& q) } \\
& \text { The car is not out of gas and it does not stail. }(\sim p \&-q) \\
& \text { None of the above is inconsistent with the rule. }
\end{aligned}
$$

The contextual cuing theories generate precise predictions regarding the effects of necessity/sufficiency relations on responses to this task. For sufficient relationships, the $p \& q$ contingency should be perceived to contradict the rule. This is because a sufficient relationship is one in which the occurrence of $p$ guarantees the occurrence of $q$; thus, the occurrence of $p$ without $q$ contradicts the rule. For necessary relationships, the $\sim p$ \& $q$ contingency should be seen as inconsistent with the rule. Because a necessary relationship is one in which the absence of $p$ guarantees the absence of $q$, a situation in which $p$ does not occur, but $q$ does, is inconsistent with the rule. Consequently, for $+\mathrm{N}+\mathrm{S}$ statements, both the $\mathrm{p}$ $\& \sim q$ and $\sim p \& q$ contingencies should be perceived to contradict the conditional rule. For $-\mathrm{N}+\mathrm{S}$ relationships, the $p \& \sim q$ contingency should be perceived as inconsistent, and for $+\mathrm{N}-\mathrm{S}$ statements, the $\sim \mathrm{p} \& \mathrm{q}$ contingency should be seen to contradict the rule. For $-\mathrm{N}-\mathrm{S}$ relationships, the "none of the above" option should be chosen. 
These response patterns are logically related to the response patterns discussed in Experiment 1. Specifically, making the $p \& \sim q$ response is logically equivalent to making the MP and MT inferences, and the $\sim p \&$ q response corresponds to the DA and AC inferences. In addition, the mapping between forward and reverse forms is conceptually similar to that observed in Experiment 1. Specifically, the content of the forward $p \& \sim q$ and reverse $\sim p \& q$ responses is the same, as is the content of the forward $\sim p \& q$ and reverse $p \& \sim q$ responses.

\section{Method}

Subjects

Forty undergraduate students from the University of Western Ontario participated to fulfill a course requirement in an introductory psychology course. One subject's data were discarded because the subject failed to follow instructions.

\section{Materials}

The 32 forward sentences used in Experiment 1, as well as their reversed counterparts, constituted the materials for this experiment. For each of these 64 sentences, four scenarios corresponding to the $p \& q, p \& \sim q, \sim p \& q$, and the $\sim p \& \sim q$ contingencies were constructed in the manner illustrated by the example above.

\section{Procedure}

Apart from the exceptions noted below, the procedure for this experiment was the same as for Experiment 1. As in Experiment 1, the conditional sentences were presented seven to a page. For each question, the conditional statement was underlined, and was followed by the four $p$ and $q$ scenarios. These scenarios were presented in a different random order for every item. Following the four scenarios was the statement, "None of the above are inconsistent with the rule." The counterbalancing of the conditional statements was the same as in Experiment 1.

The instructions explained that the purpose of the study was to examine deductive reasoning. Of the four scenarios that followed each conditional rule, the subjects were told to indicate only those which contradicted the rule. If none of the scenarios were contradictory, they were to select the "none of the above" option. The subjects were told to choose the appropriate scenarios only on the basis of conclusions that followed logically from the original statement.

\section{Results and Discussion}

\section{"Error" Responses}

Neither the $p \& q$ scenario nor the $\sim p \& \sim q$ scenario is inconsistent with either necessary or sufficient relationships; consequently, neither response was expected to vary systematically as a function of perceived necessity and sufficiency. Overall, the percentage of subjects selecting these responses was too low $(11 \%$ and $8 \%$, respectively) to satisfy the assumptions of normality and heterogeneity of variance required for the ANOVA. However, although statistical analysis of these responses was not possible, there did not seem to be any systematic variability in the percentage of people who selected each response. For the $p \& q$ responses, the difference in overall selection rates between forward and reversed forms was only $1 \%$, and the range of values among the four NS conditions was between $10 \%$ and $12 \%$ for forward statements, and between $10 \%$ and $11 \%$ for reverse statements. Similarly, for the $\sim$ p \& $\sim q$ responses, there was no difference between forward and reverse forms, and the range of values among the NS cells was $3 \%$ for the forward statements and $4 \%$ for the reverse statements.

\section{Forward Sentences: Necessity and Sufficiency by Pragmatic Relation}

As was the case in Experiment 1, only the forward statements were used to test the extent to which the effects of perceived necessity and sufficiency generalized across pragmatic relations. The following sections summarize the analyses of the $p \& \sim q$ and $\sim p \& q$ responses. Because the "none of the above" option was chosen on only $7 \%$ of all trials, these responses were not analyzed in detail. However, consistent with the contextual cuing theories, these responses appeared to be more common for nonsufficient than for sufficient relations ( $13 \%$ vs. $1 \%)$. In addition, selection of the "none" option seemed to vary by pragmatic relation. For the nonsufficient relations,
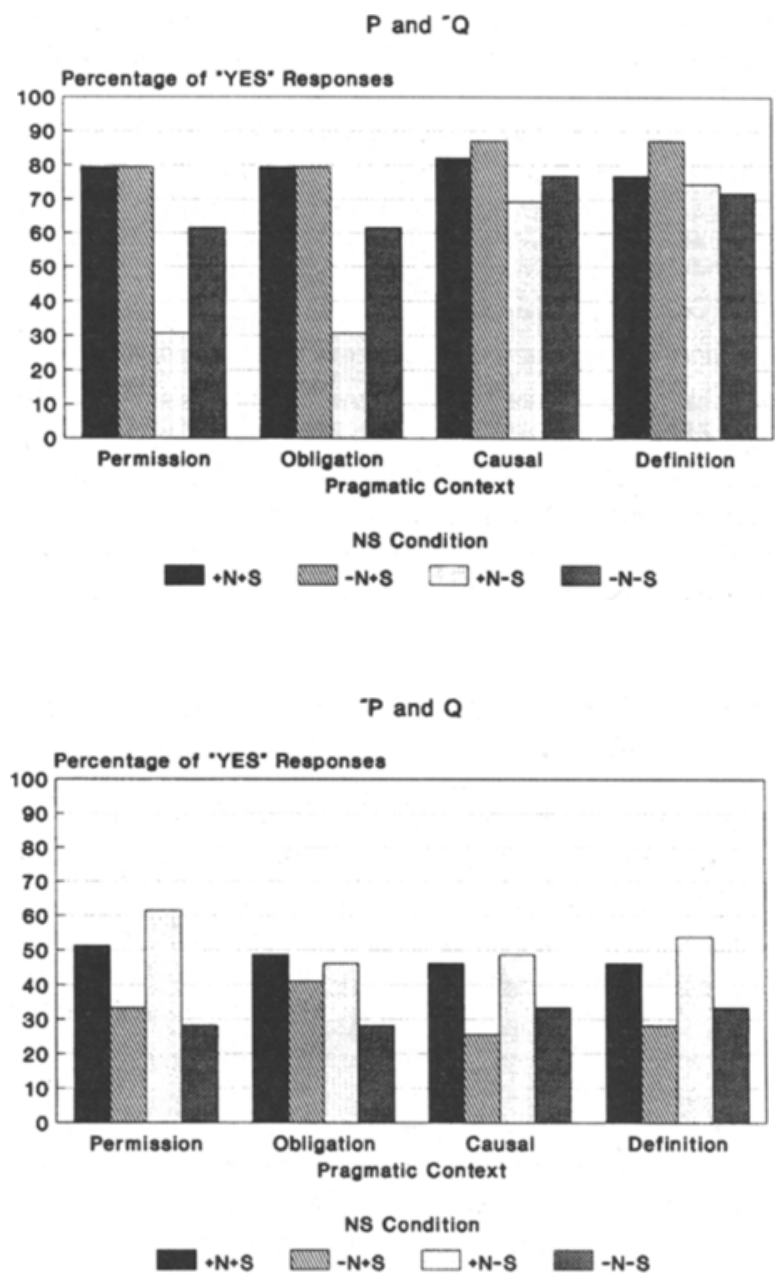

Figure 4. Percentage of $p \& \sim q$ and $p \& q$ responses, as a function of necessity, sufficiency, and pragmatic relation. NS, necessity/sufficiency: $+N$, necessary; $+S$, sufficient; $-N$, nonnecessary; $-S$, nonsufficient. 
permission and obligation statements were more likely to elicit the "none" response than either causal or definition statements ( $22 \%$ vs. $8 \%$ ).

The percentage of subjects indicating that the $p \& \sim q$ and $\sim p$ \& $q$ scenarios contradicted the conditional rules in each necessity/sufficiency $\times$ pragmatic relation condition are summarized in Figure 4. These data were analyzed with three-factor (pragmatic relation $\times$ necessity $\times$ sufficiency) within-subjects ANOVAs. Source tables for the ANOVAs are presented in Appendix C. The results of these analyses are discussed below; the $p \& \sim q$ responses are analyzed first, followed by the $p$ \& \& $q$ responses.

p \& $\sim \mathbf{q}$ responses. Analysis of $p \& \sim q$ responses revealed a significant three-factor interaction between necessity, sufficiency, and pragmatic relation. Consequently, in order to examine the effects of necessity and sufficiency for each pragmatic relation, the percentage of $p \& \sim q$ selections for each relation was analyzed with a two-factor (necessity $\times$ sufficiency) ANOVA. Consistent with the contextual cuing theories, people were more likely to choose the $\mathrm{p} \& \sim \mathrm{q}$ scenario for sufficient than nonsufficient relations $[F(1,38) \geq 6.11, p \leq .018]$ for all but the definition statements $[F(1,38)=3.03$, $p=.09]$. These findings, generally consistent with those of Experiment 1, confirm that the effects of perceived sufficiency generalize across both tasks and pragmatic relations.

In addition, there was a significant effect of necessity $[F(1,38) \geq 6.91, p \leq .012]$, as well as a significant necessity $\times$ sufficiency $[F(1,38) \geq 6.91, p \leq .012]$ for the permission and obligation statements. The pattern for these responses is similar to the pattern observed for the MP and MT responses in Experiment 1: there was an especially low rate of $\mathrm{p} \& \sim \mathrm{q}$ choices in the $+\mathrm{N}-\mathrm{S}$ condition (Newman-Keuls, $\alpha=.05$ ). Given the similarity in the overall pattern, these effects can probably be attributed to the same factors that affected the MP and MT responses in this condition.

$\sim p \& q$ responses. The analysis of the $\sim p$ \& q choices was more straightforward, because none of the interaction terms from the three-factor analysis was significant (see Appendix C). Thus, the main effects can be easily interpreted: there was no effect of pragmatic relation or sufficiency, but there was a large effect of perceived necessity. Thus, as is consistent with the contextual cuing theories, once all other factors have been equated, instances of $\sim p \& q$ were seen to violate necessary rules more often than nonnecessary rules.

Summary. These findings replicate the findings of Experiment 1: responses on the truth table task varied predictably as a function of the perceived necessity and sufficiency of the conditional relationship for all the pragmatic relations tested here. Collectively, the findings of these two experiments suggest that effects of perceived necessity and sufficiency are not restricted to a subset of pragmatic relations; instead, it would appear that necessity/sufficiency relations are relevant to the interpreta- tion of both social relations (i.e., permission and obligation) and physical relations (i.e., causation and definition).

\section{Analysis of the Reversed Statements}

The percentage of people who indicated that the $p \&$ $\sim q$ and $\sim p$ \& q scenarios contradicted the conditional rule is summarized in Figure 5 as a function of necessity, sufficiency, and temporal sequence; questions having the same content, but different syntactic forms, are vertically aligned.

Recall that by reversing the conditional statement, the syntactic forms of the logical questions were changed. Thus, the $p \& \sim q$ scenario for the forward sentences had the same content as the $\sim p \&$ q scenario for the reverse sentences; similarly, the $\sim p$ \& q scenarios for the forward sentences had the same content as the $p \& \sim q$ scenarios for the reverse sentences. Thus, if responses were based on the content of the questions, then responses to the re-
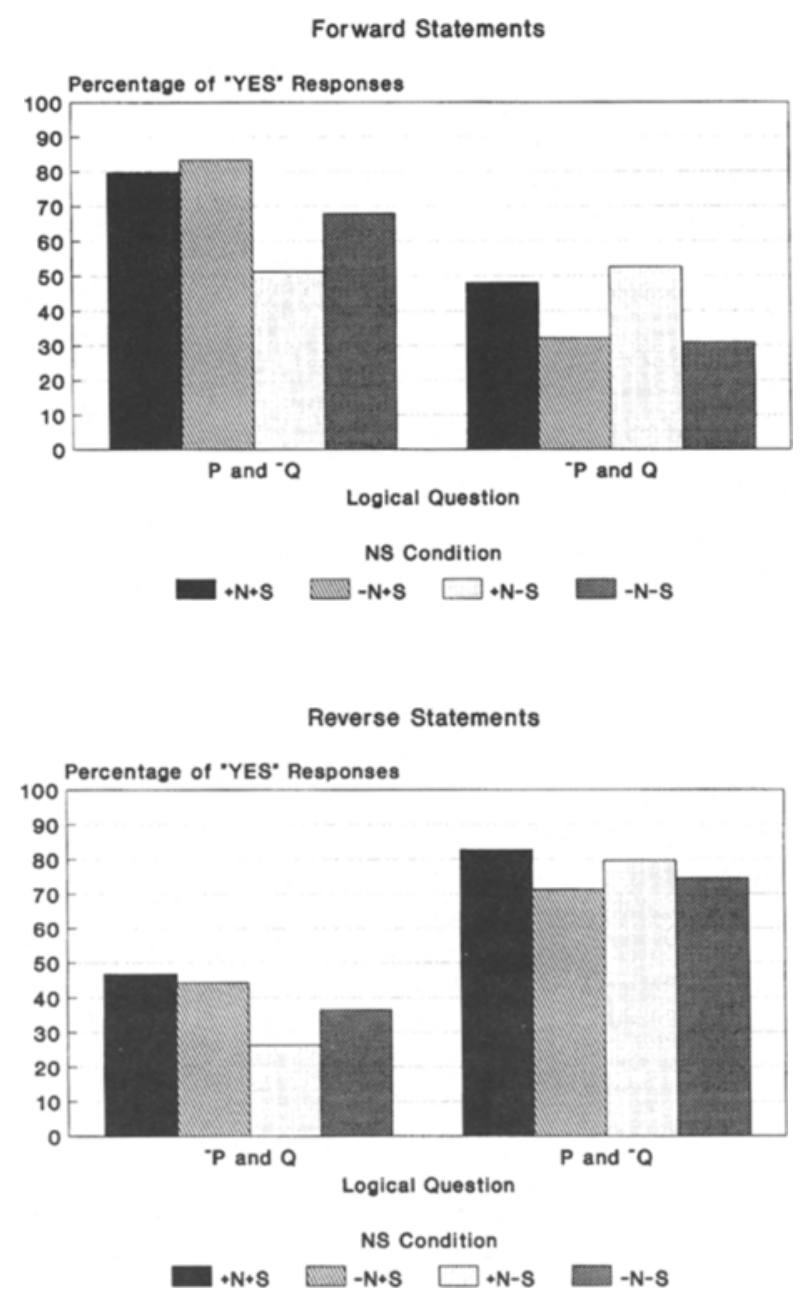

Figure 5. Percentage of $p \& \sim q$ and $p \& q$ responses, as a function of necessity, sufficiency, and syntactic form. NS, necessity/sufficiency: $+N$, necessary; $+S$, sufficient; $-\mathbf{N}$, nonnecessary; $-\mathbf{S}$, nonsufficient. 
versed scenarios should be mediated by the same factors that predicted responses to their forward counterparts. In addition, if responses are mediated by syntactic relations, then subjects should be more likely to select the logically correct $p \& \sim q$ scenario than its $\sim p \& q$ counterpart. Two sets of analyses tested these hypotheses. Source tables for these analyses are presented in Appendix C.

Forward $p \& \sim q$ versus reversed $\sim p \& q$ responses. For the first analysis, a three-factor (necessity $X$ sufficiency $X$ syntactic form) within-subjects ANOVA compared the percentage of people who indicated that the $p \&$ \& scenario was inconsistent with the forward statements with the percentage of people who indicated that the $-p \& q$ scenario was inconsistent with the reversed statements. As expected, there was a main effect of sufficiency, such that the forward $p \& \sim q$ and reverse $\sim p \& q$ responses were selected more often for sufficient than for nonsufficient relationships. In addition, there was a main effect of necessity, and a necessity $\times$ sufficiency interaction. As in previous analyses, this interaction can be localized in the $+\mathrm{N}-\mathrm{S}$ condition.

The effects of syntactic form are more salient in this analysis than they were in Experiment 1. There was an overall effect of syntactic form, and despite the interaction between syntactic form and sufficiency, paireddifference $t$ tests $(\alpha=.05 / 4)$ indicated that the logically valid $p \& \sim q$ response was chosen more often than its reverse $\sim p \&$ q counterpart in each NS condition. Finally, because of the observed interaction between sufficiency and syntactic form, separate two-factor (necessity $x$ sufficiency) ANOVAs were computed for the forward and reversed sentences; there was a significant effect of sufficiency for both the forward $\mathrm{p} \& \sim \mathrm{q}$ responses $\left[F(1,38)=33.27, M S_{\mathrm{e}}=556.89, p<.001\right]$ and the reverse p \& q responses $\left[F(1,38)=14.04, M S_{\mathrm{e}}=552.46\right.$, $p=.001]$. Consequently, the interaction between sufficiency and syntactic form should be interpreted to indicate that selection of the reverse $\sim p$ \& q scenario was less affected by the sufficiency of the relationship than was selection of the forward $p \& \sim q$ scenario. These data support the conclusion that responses on this task were mediated by both the content of the question and the syntactic form of the question.

Forward $\sim p \& q$ versus reversed $p \& \sim q$ responses. A similar conclusion was reached from the comparison of the forward $\sim p \& q$ responses to their reverse $p \& \sim q$ counterparts. Because of the significant interaction between necessity and syntactic form, further analyses were required for interpretation of the main effects of necessity and syntactic form. Paired-difference $t$ tests $(\alpha=$ $.05 / 4)$ indicated that people were more likely to choose the logically consistent $p \& \sim q$ scenario than its $\sim p \& q$ counterpart in all four NS conditions. In addition, separate two-factor (necessity $x$ sufficiency) ANOVAs indicated that selection of the forward $\sim p$ \& q responses $\left[F(1,38)=23.27, M S_{\mathrm{e}}=599.27, p<.001\right]$ and the reverse $\mathrm{p} \& \sim \mathrm{q}$ responses $\left[F(1,38)=5.46, M S_{\mathrm{e}}=496.16\right.$, $p<.025]$ both varied as a function of the necessity of the conditional relationship. Thus, the interaction can be taken to mean that necessity had less of an effect on the reverse $p \& \sim q$ selections than the forward $\sim p \& q$ selections.

Summary. Perceived necessity and sufficiency affected response patterns for both forward and reversed statements, supporting the role of content-based interpretations in reasoning. However, as was the case in Experiment 1 , the syntactic form of the question was also important. As is consistent with formal logic, people were more likely to indicate that the $p \& \sim q$ scenario contradicted the rule than they were its content-similar $\sim p \&$ $q$ counterpart. Finally, there was evidence that a third factor influenced response patterns: The differences among the NS cells were smaller for reversed than for forward sentences. This finding suggests that some additional interpretational factor associated with the reversed sentences overrode subjects' use of necessity/sufficiency information. Moreover, this effect was not associated with syntactic form per se, because it was observed for both the reversed $p \& \sim q$ as well as the reversed $\sim p \&$ q responses.

\section{GENERAL DISCUSSION}

The findings presented here have several implications for theories of interpretational and inferential processes in conditional reasoning. Specifically, these data address questions concerning (1) which factors are important to the interpretation of conditional relationships, (2) how these factors interact with each other, and (3) how interpreted information interacts with logical and syntactic processes.

\section{Necessity, Sufficiency, and the Contextual Cuing Theories}

Consistent with the contextual cuing theories, perceived necessity and sufficiency relations had robust, predictable effects on performance on two reasoning tasks. On the conditional arguments task, subjects were more likely to give "yes" and "no" responses to the MP and MT questions for sufficient than for nonsufficient relations, and they were more likely to give "maybe" responses to nonnecessary than to necessary relations. On the truth table task, subjects indicated that occurrences of $p \& \sim q$ violated sufficient relations more often than nonsufficient relations, and that occurrences of $\sim p \& q$ violated necessary relations more often than nonnecessary relations. Collectively, these findings offer strong support for the utility of necessity/sufficiency relations in predicting conditional inference patterns.

\section{Necessity, Sufficiency, and Pragmatic Relations}

The data presented here did not support initial speculations that necessity/sufficiency information would be useful for interpreting some pragmatic relations, but not others: perceived necessity/sufficiency predicted responses to both social (permission and obligation) as well as physical (definition and causal) relations. Moreover, even though the different pragmatic relations presumably cued different types of counterexamples, the effects of perceived necessity and sufficiency were still 
observed for all the pragmatic relations tested. This finding suggests that the presence or absence of alternative antecedents and consequents per se can determine the acceptability of inferences, regardless of whether these counterexamples instantiate "rule violators" or "alternative causes."

However, it is possible that differences in counterexamples could interact with task demands in such a way that differences relatively unimportant to some tasks, such as the conditional arguments and truth tables task, might be crucially important to other tasks, such as the Wason selection task. On the Wason four-card selection task, subjects are required to discover potentially falsifying cases to the conditional rule; some types of counterexamples may be more helpful to this process than others. For example, if counterexamples to the rule could be interpreted in terms of "cheaters" or other types of rule violations, they may cue a strategy, such as the "look for cheaters" strategy, that directs attention to falsifying instances (Cosmides, 1989; Gigerenzer \& Hug, 1992). Thus, whereas differences in counterexamples may be of relatively small importance for determining whether an argument is acceptable, as is required by the conditional arguments task, these differences may be very salient for identifying falsifying cases, as is required by the Wason task.

\section{Necessity, Sufficiency, and Temporal Sequence}

The interactions between necessity, sufficiency, and temporal sequence observed in Experiment 2 indicated that the effects of both necessity and sufficiency were reduced for reversed relative to forward sentences. Moreover, this finding was observed for both the $p \& \sim q$ as well as the $\sim p$ \& $q$ syntactic forms. Similar interactions were also observed when the forward $\mathrm{AC}$ and DA responses were compared with their reversed MP and MT counterparts in Experiment 1, although interpretation of those findings was difficult, given the presence of floor effects. These interactions indicate that interpretations based on the temporal relationship of the antecedent and consequent events per se can override or reduce the importance of interpretations based on necessity/sufficiency information.

One possible explanation for this result concerns the nature of the relationship expressed by the forward and reversed sentences. As illustrated below, whereas inferences based on forward sentences are primarily about the relationship between two events, inferences about reversed sentences are based primarily on epistemic relationships--that is, on relationships between two states of knowledge (Fillmore, 1987; Gibbard, 1980). For example, the forward statement below clearly implies that the consequent event (flag at half mast) is contingent upon the antecedent event (someone important dying). However, in the case of the reversed sentence, the contingency expressed is between two states of knowledge: Knowing that the flag is at half mast is grounds for concluding that someone important has died.
Forward:

If someone important dies, then the flag is flown at half mast.

Reversed:

If the flag is at half mast, then someone important has died.

This difference between event and epistemic relations potentially explains the interactions between necessity/ sufficiency and temporal sequence. Specifically, because the measures of necessity and sufficiency used here measured the necessity and sufficiency of one event for another, they may not have been as useful in predicting inferences about epistemic contingencies. Other types of measures, specifically designed to measure the necessity and sufficiency of epistemic relationships, might have been better suited to predicting inference patterns with the reversed sentences.

\section{Necessity, Sufficiency, and Syntax}

The findings from both experiments indicated that necessity/sufficiency relations predicted response patterns for all syntactic forms. Similarly, syntactic form also affected responses for all necessity/sufficiency relations. These data support two main conclusions regarding the relationship between interpretational and inferential processes.

First, they suggest that performance on the arguments and truth-tables task was not mediated only by contentbased factors. Instead, these data are consistent with the supposition that conditional relationships are represented at an abstract level, and that this abstract representation has a constant form regardless of the content of the conditional statement. Current theorizing on this issue proposes that this abstraction represents the contingent relationship between the antecedent and consequent, either as an inference rule (Braine \& O'Brien, 1991) or as a mental model (Johnson-Laird et al., 1992). Although both approaches postulate that the initial representation is little more than a license to make the MP inference, they also postulate more complex procedures for making MT inferences that are derived from this representation. Consequently, these theories suggest that both the MP and MT inferences are mediated to some extent by content-free factors thät do not mediate either the DA or the AC inferences. These assumptions suffice to explain why subjects were more likely to give determinant responses to the MP and MT questions than to the $\mathrm{AC}$ and DA questions, even when the content of the former questions was the same as the content of the latter questions.

Second, these findings also suggest that the processes by which the content of an inference is evaluated and interpreted are, to some extent, independent of the form of that inference. That is, interpretations based on necessity/ sufficiency relations appeared to operate in qualitatively similar ways for all syntactic relations. This suggests that content-based interpretations of conditional relations are represented and evaluated regardless of the 
syntactic structure of the inferences that is being considered. Indeed, these content-based interpretations can override considerations derived from syntactic form: even the straightforward MP inference varied greatly as a function of the perceived sufficiency of the conditional relationship (see Evans, Barston, \& Pollard, 1983; Newstead, Pollard, Evans, \& Allen, 1992; Oakhill, JohnsonLaird, \& Garnham, 1989, for a related discussion).

\section{Conclusions}

The emphasis in this paper has been on the role of contextual and interpretive factors in reasoning. Together with previous findings (e.g., those of Cummins et al., 1991; Markovits, 1984; Markovits \& Nantel, 1989; Thompson, in press), these data suggest that preexisting knowledge exerts a powerful influence on the inferences people make, even to the extent of overriding considerations based on logical validity. Moreover, the interactions observed between problem content and syntax stress the importance of developing a well-formulated model of how the interpretive phase of reasoning is integrated with the underlying inferential processes. Indeed, the absence of such a theory has important implications for current models of conditional reasoning. Specifically, without a well-developed explanation of interpretation, reasoning theories become overly powerful and untestable, because any unexplained variability can always be attributed to either the working or the failure of an "interpretive" component. Thus, in order to escape circularity, it is important to determine which factors can influence the interpretation of conditional relationships, and to specify the relationship between these interpretations and the processes used to make and evaluate inferences.

\section{REFERENCES}

Bindra, D., Clarke, K. A., \& Schultz, T. R. (1980). Understanding predictive relations of necessity and sufficiency in formally equivalent "causal" and "logical" problems. Journal of Experimental Psychology: General, 109, 422-443.

Braine, M. D. S., \& O'Brien, D. P. (1991). A theory of If: A lexical entry, reasoning program, and pragmatic principles. Psychological Review, 98, 182-203.

Bucci, W. (1978). The interpretation of universal affirmative propositions. Cognition, 6, 55-77.

BYRNE, R. M. J. (1989). Suppressing valid inferences with conditionals. Cognition, 31, 61-83.

Cheng, P. W., \& Holyoak, K. J. (1985). Pragmatic reasoning schemas. Cognitive Psychology, 17, 391.416.

Cheng, P. W., HolyoaK, K. J., Nisbett, R. E., \& Oliver, L. M. (1986). Pragmatic versus syntactic approaches to training deductive reasoning. Cognitive Psychology, 18, 293-328.

Cosmides, L. (1989). The logic of social exchange: Has natural selection shaped how we reason? Studies with the Wason selection task. Cognition, 31, 187-276.

Cummins, D. D., Lubart, T., Alksnis, O., \& Rist, R. (1991). Conditional reasoning and causation. Memory \& Cognition, 19, 274-282.

DigDon, N. L. (1986). Conditional and biconditional interpretations of if-then sentences: The role of content and context. Unpublished doctoral dissertation, University of Western Ontario.

Evans, J. ST. B. T. (1982). The psychology of deductive reasoning. London: Routledge \& Kegan Paul

Evans, J. ST. B. T. (1983). Linguistic determinants of bias in conditional reasoning. Quarterly Journal of Experimental Psychology, 35A, 635- 644 .
Evans, J. St. B. T., Barston, J. L., \& Pollard, P. (1983). On the conflict between logic and belief in syllogistic reasoning. Memory \& Cognition, 11, 295-306.

Fillenbaum, S. (1986). The use of conditionals in deterrents and inducements. In E. C. Traugott, A. T. Meullen, J. S. Reilly, \& C. A. Ferguson (Eds.), On conditionals (pp. 179-195). Cambridge: Cambridge University Press.

Fillmore, C. J. (1987). Varieties of conditional sentences, In Proceedings of the 3rd Eastern States Conference on Linguistics (pp. 163-182). Columbus: Ohio State University.

GibBard, A. (1980). Two recent theories of conditionals. In W. L. Harper and G. Pearce (Eds.), Ifs (pp. 211-247). Dordrecht: D. Reidel.

GigerEnZER, G., \& HUG, K. (1992). Domain-specific reasoning: Social contracts, cheating, and perspective change. Cognition, 43, 127 171.

GrIGGS, R. A. (1983). The role of problem content in the selection task and in the THOG problem. In J. St. B. T. Evans (Ed.), Thinking and reasoning (pp. 16-43). London: Routledge \& Kegan Paul.

Johnson-LaIRD, P. N., Byrne, R. M. J., \& Schaeken, W. (1992). Propositional reasoning by model. Psychological Review, 99, 418439.

MARCUS, S. L., \& RIPS, L. J. (1979). Conditional reasoning. Journal of Verbal Learning \& Verbal Behavior, 18, 199-223.

Markovits, H. (1984). Awareness of the 'possible' as a mediator of formal thinking in conditional reasoning problems. British Journal of Psychology, 75, 367-376.

MarKovits, H. (1986). Familiarity effects in conditional reasoning. Journal of Educational Psychology, 78, 492-494.

Markovits, H., \& Nantel, G. (1989). The belief-bias effect in the production and evaluation of logical conclusions. Memory \& Cognition, 17, 11-17.

Markovits, H., \& Savary, F. (1992). Pragmatic schemas and the selection task: To reason or not to reason. Quarterly Journal of Experimental Psychology, 45A, 133-148.

Newstead, S. E., Pollard, P., Evans, J. St. B. T., \& Allen, J. L. (1992). The source of belief bias effects in syllogistic reasoning. Cognition, 45, 257-284.

OAKhill, J., Johnson-Laird, P. N., \& Garnham, A. (1989). Believability and syllogistic reasoning. Cognition, 31, 117-140.

PollaRd, P. (1982). Human reasoning: Some possible effects of availability. Cognition, 12, 65-96.

REICH, S. S., \& RUTH, P. (1982). Wason's selection task: Verification, bias and matching. British Journal of Psychology, 73, 395-405.

Rumaine, B., Connell, J., \& Braine, M. D. S. (1983). Conversational comprehension processes are responsible for reasoning fallacies in children as well as adults: If is not the biconditional. Developmental Psychology, 19, 471-481.

STAUDENMAYER, H. (1975). Understanding conditional reasoning with meaningful propositions. In R. J. Falmagne (Ed.), Reasoning: Representation and process (pp. 55-79). New York: Wiley.

TAPLIN, J. E. (1971). Reasoning with conditional sentences. Journal of Verbal Learning \& Verbal Behavior, 10, 219-225.

TAPLIN, J. E., \& STAUDENMAYER, H. (1973). Interpretation of abstract conditional sentences in deductive reasoning. Journal of Verbal Learning \& Verbal Behavior, 12, 530-542.

THOMPSON, V. A. (in press). Conditional reasoning: The necessary and sufficient conditions. Canadian Journal of Experimental Psychology.

WARD, S. L., BYRNES, J. P., \& OVERTON, W. F. (1990). Organization of knowledge and conditional reasoning. Journal of Educational Psychology, 82, 832-837.

\section{NOTES}

1. Note that in order to develop both sufficient and nonsufficient permission relations, it was necessary to create two types of normative ratings questions for the permission items. The first dealt with acquired rights, and the second dealt with desired actions. Take the drinking-age rule, for example (i.e., if a customer is drinking alcohol, then he/she must be over 19). In this case, being over 19 is sufficient to have the right to drink, but it is not sufficient to guarantee that someone will have a drink. Thus, whether or not the relation is sufficient 
depends on whether one thinks in terms of actions or rights. The normative questions reflected this property of permission statements: those in the +sufficiency cells addressed acquired rights, and those in the - sufficiency cells addressed desired actions.
2. For the permission statements, the form of the logical questions paralleled the ratings questions. Thus, if the ratings questions addressed acquired rights, so did the logical questions. Conversely, if the ratings questions addressed desired actions, so did the logical questions.

\section{APPENDIX A}

The following 32 statements are the items used in Experiments 1 and 2. An "a" preceding an item designates the forward temporal sequence; $a$ " $b$," the reverse temporal sequence. The " $R$ " indicates items for which the questions were based on acquired rights.

\begin{tabular}{|c|c|c|c|}
\hline NS Type & $\begin{array}{c}\text { Pragmatic } \\
\text { Context }\end{array}$ & $\begin{array}{l}\text { Temporal } \\
\text { Sequence }\end{array}$ & Statement \\
\hline \multirow[t]{16}{*}{$+\mathrm{N}+\mathrm{S}$} & \multirow[t]{4}{*}{ Causal } & a & If butter is heated, then it melts. \\
\hline & & $\mathrm{b}$ & If butter melts, then it has been heated. \\
\hline & & a & If water is heated to 100 degrees centigrade, then it boils. \\
\hline & & b & If water boils, then it has been heated to 100 degrees centigrade. \\
\hline & \multirow[t]{4}{*}{ Permission } & a & $\begin{array}{l}\text { If the planning board gives its approval, then an owner has the right to change his } \\
\text { building to commercial use. (R) }\end{array}$ \\
\hline & & b & $\begin{array}{l}\text { If an owner has the right to change his building to commercial use, then the planning } \\
\text { board has given its approval. (R) }\end{array}$ \\
\hline & & a & $\begin{array}{l}\text { If the licensing board grants them a license, then a restaurant is allowed to sell liquor. } \\
\text { (R) }\end{array}$ \\
\hline & & b & $\begin{array}{l}\text { If a restaurant is allowed to sell liquor, then the licensing board has granted them a } \\
\text { license. (R) }\end{array}$ \\
\hline & \multirow[t]{4}{*}{ Definition } & a & If an animal is warm-blooded, then it is a mammal. \\
\hline & & $\mathrm{b}$ & If an animal is a mammal, then it is warm-blooded. \\
\hline & & a & $\begin{array}{l}\text { If a person is someone's mother's mother, then she is that person's maternal grand- } \\
\text { mother. }\end{array}$ \\
\hline & & $\mathrm{b}$ & $\begin{array}{l}\text { If a person is someone's maternal grandmother, then she is that person's mother's } \\
\text { mother. }\end{array}$ \\
\hline & \multirow[t]{4}{*}{ Obligation } & a & $\begin{array}{l}\text { If a Canadian citizen is over } 65 \text {, then the government must pay them an old age pen- } \\
\text { sion. }\end{array}$ \\
\hline & & $\mathrm{b}$ & $\begin{array}{l}\text { If the government must pay a Canadian citizen an old age pension, then they are } \\
\text { over } 65 \text {. }\end{array}$ \\
\hline & & a & $\begin{array}{l}\text { If an injured person arrives at "Emergency," then the emergency staff must treat } \\
\text { them. }\end{array}$ \\
\hline & & $\mathrm{b}$ & $\begin{array}{l}\text { If the emergency staff must treat someone, then an injured person has arrived at "Emer- } \\
\text { gency." }\end{array}$ \\
\hline \multirow[t]{12}{*}{$-\mathrm{N}+\mathrm{S}$} & \multirow[t]{4}{*}{ Causal } & a & If the car is out of gas, then it stalls. \\
\hline & & $b$ & If the car stalls, then it is out of gas. \\
\hline & & a & If the dog tracks mud on the floor, then the floor is dirty. \\
\hline & & $\mathrm{b}$ & If the floor is dirty, then the dog has tracked mud on it. \\
\hline & \multirow[t]{4}{*}{ Permission } & a & $\begin{array}{l}\text { If a person has a PhD in astrophysics, then they are qualified to teach at a univer- } \\
\text { sity. (R) }\end{array}$ \\
\hline & & $\mathrm{b}$ & $\begin{array}{l}\text { If a person is qualified to teach at a university, then they have a } \mathrm{PhD} \text { in astrophysics. } \\
\text { (R) }\end{array}$ \\
\hline & & a & $\begin{array}{l}\text { If a person has a valid Visa card, then they are able to make a purchase in a department } \\
\text { store. }(R)\end{array}$ \\
\hline & & b & $\begin{array}{l}\text { If a person is able to make a purchase in a department store, then they have a valid } \\
\text { Visa card. }(R)\end{array}$ \\
\hline & \multirow[t]{4}{*}{ Definition } & a & If an animal is a robin, then it is a bird. \\
\hline & & $\mathrm{b}$ & If an animal is a bird, then it is a robin. \\
\hline & & a & If a card has a jack on it, then it is a face card. \\
\hline & & $\mathrm{b}$ & If a card is a face card, then it has a jack on it. \\
\hline
\end{tabular}




\begin{tabular}{|c|c|c|c|}
\hline \multicolumn{4}{|r|}{ APPENDIX A (Continued) } \\
\hline NS Type & $\begin{array}{c}\text { Pragmatic } \\
\text { Context }\end{array}$ & $\begin{array}{l}\text { Temporal } \\
\text { Sequence }\end{array}$ & Statement \\
\hline & Obligation & a & If a person is a nurse, then they must care for the sick. \\
\hline & & b & If a person must care for the sick, then they are a nurse. \\
\hline & & a & $\begin{array}{l}\text { If a urithium miner gets throat cancer, then the company must pay him compensa- } \\
\text { tion. }\end{array}$ \\
\hline & & b & $\begin{array}{l}\text { If the company must pay a urithium miner compensation, then he has gotten throat } \\
\text { cancer. }\end{array}$ \\
\hline \multirow[t]{16}{*}{$+N-S$} & Causal & a & If the T.V. is plugged in, then it works. \\
\hline & & $\mathrm{b}$ & If the T.V. works, then it is plugged in. \\
\hline & & a & If the car has gas in it, then it runs. \\
\hline & & b & If the car runs, then it has gas in it. \\
\hline & Permission & a & If a person is over 19, then they are allowed to drink in bars. \\
\hline & & b & If a person is allowed to drink in bars, then they are over 19 \\
\hline & & a & $\begin{array}{l}\text { If an athlete passes the drug test at the Olympics, then the IOC can give them a } \\
\text { medal. }\end{array}$ \\
\hline & & b & $\begin{array}{l}\text { If the IOC can give an athlete a medal, then they have passed the drug test at the } \\
\text { Olympics. }\end{array}$ \\
\hline & Definition & a & If a plant has roots, then it is a tree. \\
\hline & & $\mathrm{b}$ & If a plant is a tree, then it has roots. \\
\hline & & a & If a figure has 4 sides, then it is a square. \\
\hline & & b & If a figure is a square, then it has 4 sides. \\
\hline & Obligation & a & If a person is summoned for jury duty, then they might have to sit on a jury. \\
\hline & & b & If a person might have to sit on a jury, then they have been summoned for jury duty. \\
\hline & & a & $\begin{array}{l}\text { If a student registers in a course, then the university might have to give them credit } \\
\text { for the course. }\end{array}$ \\
\hline & & b & $\begin{array}{l}\text { If the university might have to give a student credit for a course, then they have reg- } \\
\text { istered in the course. }\end{array}$ \\
\hline \multirow[t]{16}{*}{$-\mathrm{N}-\mathrm{S}$} & Causal & a & If the weather conditions are bad, then the plane will crash. \\
\hline & & $\mathrm{b}$ & If the plane has crashed, then the weather conditions have been bad. \\
\hline & & a & If a person eats toffee, then they get cavities. \\
\hline & & b & If a person gets cavities, then they eat toffee. \\
\hline & Permission & a & If a person has taken an automotives course in high school, then they can fix cars. \\
\hline & & b & If a person can fix cars, then they have taken an automotives course in high school. \\
\hline & & a & If the light is red, then the driver can turn right. (R) \\
\hline & & b & If the driver can turn right, then the light is red. (R) \\
\hline & Definition & a & If a piece of fruit is red, then it is an apple. \\
\hline & & $\mathrm{b}$ & If a piece of fruit is an apple, then it is red. \\
\hline & & a & If a piece of furniture is made of wood, then it is a chair. \\
\hline & & $\mathrm{b}$ & If a piece of furniture is a chair, then it is made of wood. \\
\hline & Obligation & a & If a person witnesses an accident, then they should help the victim. \\
\hline & & $\mathrm{b}$ & If a person should help the victim, then they have witnessed an accident. \\
\hline & & a & $\begin{array}{l}\text { If a manufacturer produces PCB's, then they should be careful not to pollute the en- } \\
\text { vironment. }\end{array}$ \\
\hline & & $\mathrm{b}$ & $\begin{array}{l}\text { If a manufacturer should be careful not to pollute the environment, then he manu- } \\
\text { factures PCB's. }\end{array}$ \\
\hline
\end{tabular}




\section{APPENDIX B}

Below is a summary of the analyses reported in Experiment 1. Where the assumptions for the analysis have not been met, the degrees of freedom have been adjusted with the Geisser-Greenhouse correction. An asterisk indicates tests that were significant at $\alpha=.05$.

\begin{tabular}{|c|c|c|c|c|c|}
\hline Dependent Measure & Factor & $F$ & $d f$ & $M S_{\mathrm{e}}$ & ETA $^{2}$ \\
\hline MP: Percentage "yes" & $\begin{array}{l}\text { PragRel } \\
\mathrm{S} \\
\mathrm{N} \\
\text { PragRel } \times \mathrm{S} \\
\text { PragRel } \times \mathrm{N} \\
\mathrm{S} \times \mathrm{N} \\
\text { PragRel } \times \mathrm{S} \times \mathrm{N}\end{array}$ & $\begin{array}{r}31.16^{*} \\
211.84^{*} \\
31.95^{*} \\
29.59^{*} \\
4.06^{*} \\
29.89^{*} \\
12.81^{*}\end{array}$ & $\begin{array}{l}2,82 \\
1,37 \\
1,37 \\
2,87 \\
3,111 \\
1,37 \\
2,92\end{array}$ & $\begin{array}{r}1,005.66 \\
1,213.10 \\
1,042.41 \\
1,029.37 \\
695.09 \\
925.05 \\
572.99\end{array}$ & $\begin{array}{l}.46 \\
.85 \\
.46 \\
.44 \\
.10 \\
.45 \\
.26\end{array}$ \\
\hline AC: Percentage "maybe" & $\begin{array}{l}\text { PragRel } \\
\mathrm{S} \\
\mathrm{N} \\
\text { PragRel } \times \mathrm{S} \\
\text { PragRel } \times \mathrm{N} \\
\mathrm{S} \times \mathrm{N} \\
\text { PragRel } \times \mathrm{S} \times \mathrm{N}\end{array}$ & $\begin{array}{c}.16 \\
.05 \\
114.09^{*} \\
3.32^{*} \\
.97 \\
1.03 \\
5.14^{*}\end{array}$ & $\begin{array}{l}3,111 \\
1,37 \\
1,37 \\
3,111 \\
3,111 \\
1,37 \\
3,111\end{array}$ & $\begin{array}{l}1,379.21 \\
1,383.36 \\
3,598.86 \\
1,064.49 \\
1,020.63 \\
1,018.85 \\
1,117.24\end{array}$ & $\begin{array}{l}.00 \\
.00 \\
.76 \\
.08 \\
.03 \\
.03 \\
.12\end{array}$ \\
\hline DA: Percentage "maybe" & $\begin{array}{l}\text { PragRel } \\
\mathrm{S} \\
\mathrm{N} \\
\text { PragRel } \times \mathrm{S} \\
\text { PragRel } \times \mathrm{N} \\
\mathrm{S} \times \mathrm{N} \\
\text { PragRel } \times \mathrm{S} \times \mathrm{N}\end{array}$ & $\begin{array}{c}1.44 \\
.06 \\
103.36^{*} \\
2.89^{*} \\
1.67 \\
.69 \\
8.82^{*}\end{array}$ & $\begin{array}{l}3,111 \\
1,37 \\
1,37 \\
3,111 \\
3,111 \\
1,37 \\
3,94\end{array}$ & $\begin{array}{r}1,430.77 \\
1,079.30 \\
3,111.66 \\
1,253.56 \\
969.65 \\
862.38 \\
1,145.69\end{array}$ & $\begin{array}{l}.04 \\
.00 \\
.74 \\
.07 \\
.04 \\
.02 \\
.19\end{array}$ \\
\hline MT: Percentage "no" & $\begin{array}{l}\text { PragRel } \\
\mathrm{S} \\
\mathrm{N} \\
\text { PragRel } \times \mathrm{S} \\
\text { PragRel } \times \mathrm{N} \\
\mathrm{S} \times \mathrm{N} \\
\text { PragRel } \times \mathrm{S} \times \mathrm{N}\end{array}$ & $\begin{array}{r}6.20^{*} \\
145.88^{*} \\
34.00^{*} \\
3.34^{*} \\
.27 \\
22.42^{*} \\
5.82^{*}\end{array}$ & $\begin{array}{l}2,82 \\
1,38 \\
1,38 \\
2,81 \\
2,79 \\
1,38 \\
2,75\end{array}$ & $\begin{array}{l}1,201.92 \\
1,716.43 \\
1,640.52 \\
1,103.52 \\
1,344.75 \\
2,161.77 \\
1,155.25\end{array}$ & $\begin{array}{l}.14 \\
.79 \\
.47 \\
.08 \\
.01 \\
.37 \\
.13\end{array}$ \\
\hline $\begin{array}{l}\text { Forward MP and reverse } \\
\text { AC: Percentage "yes" }\end{array}$ & $\begin{array}{l}\text { SynForm } \\
\mathrm{S} \\
\mathrm{N} \\
\text { SynForm } \times \mathrm{S} \\
\text { SynForm } \times \mathrm{N} \\
\mathrm{S} \times \mathrm{N} \\
\text { SynForm } \times \mathrm{S} \times \mathrm{N}\end{array}$ & $\begin{array}{c}24.15^{*} \\
363.23^{*} \\
32.80^{*} \\
3.82 \\
3.15 \\
25.63^{*} \\
6.51^{*}\end{array}$ & $\begin{array}{l}1,39 \\
1,39 \\
1,39 \\
1,39 \\
1,39 \\
1,39 \\
1,39\end{array}$ & $\begin{array}{l}798.14 \\
466.88 \\
332.00 \\
327.99 \\
318.38 \\
244.72 \\
320.05\end{array}$ & $\begin{array}{l}.38 \\
.46 \\
.09 \\
.07 \\
.09 \\
.40 \\
.14\end{array}$ \\
\hline $\begin{array}{l}\text { Forward MT and reverse } \\
\text { DA: Percentage "no" }\end{array}$ & $\begin{array}{l}\text { SynForm } \\
\mathrm{S} \\
\mathrm{N} \\
\text { SynForm } \times \mathrm{S} \\
\text { SynForm } \times \mathrm{N} \\
\mathrm{S} \times \mathrm{N} \\
\text { SynForm } \times \mathrm{S} \times \mathrm{N}\end{array}$ & $\begin{array}{c}2.86 \\
375.04^{*} \\
20.50^{*} \\
0.00 \\
3.37 \\
22.38^{*} \\
2.88\end{array}$ & $\begin{array}{l}1,39 \\
1,39 \\
1,39 \\
1,39 \\
1,39 \\
1,39 \\
1,39\end{array}$ & $\begin{array}{l}628.56 \\
512.37 \\
545.49 \\
311.38 \\
180.91 \\
423.56 \\
262.64\end{array}$ & $\begin{array}{l}.06 \\
.91 \\
.34 \\
.00 \\
.08 \\
.36 \\
.07\end{array}$ \\
\hline $\begin{array}{l}\text { Forward } \mathrm{AC} \text { and reverse MP: } \\
\text { Percentage "maybe" }\end{array}$ & $\begin{array}{l}\text { SynForm } \\
\mathrm{S} \\
\mathrm{N} \\
\text { SynForm } \times \mathrm{S} \\
\text { SynForm } \times \mathrm{N} \\
\mathrm{S} \times \mathrm{N} \\
\text { SynForm } \times \mathrm{S} \times \mathrm{N}\end{array}$ & $\begin{array}{c}45.72^{*} \\
0.34 \\
102.37^{*} \\
0.01 \\
51.04^{*} \\
0.82 \\
1.19\end{array}$ & $\begin{array}{l}1,39 \\
1,39 \\
1,39 \\
1,39 \\
1,39 \\
1,39 \\
1,39\end{array}$ & $\begin{array}{r}1,030.78 \\
252.40 \\
980.24 \\
334.67 \\
435.36 \\
306.96 \\
210.80\end{array}$ & $\begin{array}{l}.54 \\
.01 \\
.72 \\
.00 \\
.57 \\
.02 \\
.03\end{array}$ \\
\hline $\begin{array}{l}\text { Forward DA and reverse MT: } \\
\text { Percentage "maybe" }\end{array}$ & $\begin{array}{l}\text { SynForm } \\
\mathrm{S} \\
\mathrm{N} \\
\text { SynForm } \times \mathrm{S} \\
\text { SynForm } \times \mathrm{N} \\
\mathrm{S} \times \mathrm{N} \\
\text { SynForm } \times \mathrm{S} \times \mathrm{N}\end{array}$ & $\begin{array}{c}9.29^{*} \\
2.21 \\
111.46^{*} \\
3.57 \\
10.85^{*} \\
2.65 \\
2.16\end{array}$ & $\begin{array}{l}1,39 \\
1,39 \\
1,39 \\
1,39 \\
1,39 \\
1,39 \\
1,39\end{array}$ & $\begin{array}{r}554.02 \\
330.73 \\
1,118.66 \\
190.44 \\
327.72 \\
257.21 \\
212.21\end{array}$ & $\begin{array}{l}.19 \\
.05 \\
.74 \\
.08 \\
.22 \\
.06 \\
.05\end{array}$ \\
\hline
\end{tabular}

Note-MP, modus ponens; MT, modus tollens; AC, affirming the consequent; DA, denying the antecedent; PragRel, pragmatic relation; S, sufficiency; N, necessity; SynForm, syntactic form. 


\section{APPENDIX C}

Below is a summary of the analyses of the forward statements reported in Experiment 2 . Where the assumptions for the analysis have not been met, the degrees of freedom have been adjusted using the Geisser-Greenhouse correction. An asterisk indicates tests that were significant at $\alpha=.05$.

\begin{tabular}{|c|c|c|c|c|c|}
\hline Dependent Measure & Factor & $F$ & $d f$ & $M S_{\mathrm{e}}$ & ETA $^{2}$ \\
\hline p \& q: Percentage "yes" & $\begin{array}{l}\text { PragRel } \\
\mathrm{S} \\
\mathrm{N} \\
\text { PragRel } \times \mathrm{S} \\
\text { PragRel } \times \mathrm{N} \\
\mathrm{S} \times \mathrm{N} \\
\text { PragRel } \times \mathrm{S} \times \mathrm{N}\end{array}$ & $\begin{array}{c}8.99^{*} \\
33.27^{*} \\
25.10^{*} \\
6.33^{*} \\
1.80 \\
5.75^{*} \\
4.63^{*}\end{array}$ & $\begin{array}{l}2,93 \\
1,38 \\
1,38 \\
2,93 \\
2,84 \\
1,38 \\
3,114\end{array}$ & $\begin{array}{r}1,374.55 \\
2,227.56 \\
653.68 \\
1,099.58 \\
785.26 \\
1,114.20 \\
987.12\end{array}$ & $\begin{array}{l}.19 \\
.47 \\
.40 \\
.14 \\
.05 \\
.13 \\
.11\end{array}$ \\
\hline "p \& q: Percentage "yes" & $\begin{array}{l}\text { PragRel } \\
\mathrm{S} \\
\mathrm{N} \\
\text { PragRel } \times \mathrm{S} \\
\text { PragRel } \times \mathrm{N} \\
\mathrm{S} \times \mathrm{N} \\
\text { PragRel } \times \mathrm{S} \times \mathrm{N}\end{array}$ & $\begin{array}{c}.79 \\
.53 \\
23.27^{*} \\
1.27 \\
.97 \\
1.61 \\
.87\end{array}$ & $\begin{array}{l}3,114 \\
1,38 \\
1,38 \\
3,97 \\
2,92 \\
1,38 \\
3,114\end{array}$ & $\begin{array}{r}886.19 \\
762.48 \\
2,397.10 \\
1,257.31 \\
1,117.30 \\
804.66 \\
905.87\end{array}$ & $\begin{array}{l}.02 \\
.01 \\
.38 \\
.03 \\
.02 \\
.04 \\
.02\end{array}$ \\
\hline $\begin{array}{l}\text { Forward } p \& \sim q \\
\text { and reverse } \sim p \& q\end{array}$ & $\begin{array}{l}\text { SynForm } \\
\mathrm{S} \\
\mathrm{N} \\
\text { SynForm } \times \mathrm{S} \\
\text { SynForm } \times \mathrm{N} \\
\mathrm{S} \times \mathrm{N} \\
\text { SynForm } \times \mathrm{S} \times \mathrm{N}\end{array}$ & $\begin{array}{c}25.60^{*} \\
27.14^{*} \\
18.43^{*} \\
6.29^{*} \\
3.99 \\
13.56^{*} \\
0.00\end{array}$ & $\begin{array}{l}1,38 \\
1,38 \\
1,38 \\
1,38 \\
1,38 \\
1,38 \\
1,38\end{array}$ & $\begin{array}{r}3,129.85 \\
925.90 \\
210.44 \\
183.45 \\
200.95 \\
236.38 \\
328.95\end{array}$ & $\begin{array}{l}.40 \\
.42 \\
.33 \\
.10 \\
.14 \\
.26 \\
.00\end{array}$ \\
\hline $\begin{array}{l}\text { Forward } \sim \mathrm{p} \& \mathrm{q} \\
\text { and reverse } \mathrm{p} \& \sim \mathrm{q}\end{array}$ & $\begin{array}{l}\text { SynForm } \\
\mathrm{S} \\
\mathrm{N} \\
\text { SynForm } \times \mathrm{S} \\
\text { SynForm } \times \mathrm{N} \\
\mathrm{S} \times \mathrm{N} \\
\text { SynForm } \times \mathrm{S} \times \mathrm{N}\end{array}$ & $\begin{array}{c}30.77^{*} \\
0.38 \\
16.31^{*} \\
0.22 \\
10.50^{*} \\
0.01 \\
3.53\end{array}$ & $\begin{array}{l}1,38 \\
1,38 \\
1,38 \\
1,38 \\
1,38 \\
1,38 \\
1,38 \\
\end{array}$ & $\begin{array}{r}3,295.48 \\
132.32 \\
887.63 \\
231.00 \\
207.81 \\
141.81 \\
205.06\end{array}$ & $\begin{array}{l}.45 \\
.01 \\
.30 \\
.01 \\
.22 \\
.00 \\
.09\end{array}$ \\
\hline
\end{tabular}

Note-PragRel, pragmatic relation; $S$, sufficiency; $N$, necessity; SynForm, syntactic form.

(Manuscript received May 20, 1993;

revision accepted for publication October 21, 1993.) 